\title{
Chronische respiratorische Insuffizienz: Rolle der außerklinischen Beatmung
}

\author{
Chronic Respiratory Failure: The Role of Home Mechanical Ventilation
}

Autor

Institut

\section{W. Windisch}

Abteilung Pneumologie, Universitätsklinik Freiburg eingereicht 12.7.2010

akzeptiert nach Revision 13. 7.2010

\section{Bibliografie}

DOI http://dx.doi.org/

10.1055/s-0030-1255660

Pneumologie 2010; 64: 600-603 ๔ Georg Thieme

Verlag KG Stuttgart · New York ISSN 0934-8387

Korrespondenzadresse Prof. Dr. Wolfram Windisch Abteilung Pneumologie Universitätsklinik Freiburg Killianstraße 5

79106 Freiburg wolfram.windisch@ uniklinik-freiburg.de

\section{Zusammenfassung \\ $\nabla$}

Während in der ersten Hälfte des 20.Jahrhunderts die Negativ-Druck-Ventilation über KürassRespiratoren und eiserne Lungen vorherrschend war, markiert die Polio-Epidemie in Kopenhagen im Jahre 1952 den Wendepunkt mit Beginn der Positiv-Druck-Ventilation nach Tracheotomie. Mit der Einführung von Gesichtsmasken hat sich zudem die nichtinvasive Positiv-Druck-Ventilation seit ihren Anfängen 1985 in Deutschland mittlerweile als Routineverfahren zur LangzeitBehandlung von Patienten mit chronischer ventilatorischer Insuffizienz entwickelt. Der vorliegende Artikel gibt einen Überblick über diese Entwicklung und skizziert dabei auch die Rolle der nationalen Fachgesellschaften, insbesondere der „Deutschen Gesellschaft für Pneumologie und Beatmungsmedizin (DGP) e.V.“ sowie der „Deutschen Interdisziplinären Gesellschaft für außerklinische Beatmung (DIGAB) e.V.“.

\section{Einleitung}

Die Beatmungsmedizin ist heute fest etablierter Bestandteil der modernen Medizin, auch wenn eine klinische Anwendung erst seit wenigen Jahrzehnten Routine ist. Dabei ist die Entwicklung der Beatmungsmedizin wie bei vielleicht nur wenigen Disziplinen in der Medizin sehr wesentlich durch historische Ereignisse und Katastrophen sowie durch den Pioniergeist einzelner Wissenschaftler gekennzeichnet. So darf die verheerende Polio-Epidemie 1952/53 in Kopenhagen nicht nur als Geburtsstunde der modernen Beatmungs-, sondern durchaus auch der modernen Intensivmedizin insgesamt betrachtet werden, initiiert durch den herausragenden Pionier Björn Ibsen. Seine (soweit bekannte) persönliche Biografie, sein beruflicher Werdegang sowie die sich über-

\section{Abstract \\ $\nabla$}

While negative pressure ventilation using cuirass respirators or iron-lung machines was prevailing in the first part of the $20^{\text {th }}$ century, the polio epidemic in Copenhagen 1952 marks the turning point at which positive pressure ventilation following tracheotomy was started. Furthermore, following the introduction of facial masks and starting 1985 in Germany non-invasive positive pressure ventilation has meanwhile been developed as a routine procedure for the long-term treatment of patients with chronic ventilatory failure today. The current article provides an overview of these developments and also outlines the role of two particular national societies: "Deutsche Gesellschaft für Pneumologie und Beatmungsmedizin (DGP) e.V." (German Medical Association of Pneumology and Ventilatory Support) and "Deutsche Interdisziplinäre Gesellschaft für außerklinische Beatmung (DIGAB) e.V." (German Interdisciplinary Society for Home Mechanical Ventilation).

schlagenden Ereignisse um den Zeitpunkt der ersten Positiv-Druck-Beatmung der Polio-Patientin Vivi Ebert herum sind bewegend und motivierend, erzwingen aber auch eine Nachdenklichkeit, die heute unter dem Blickwinkel der scheinbar unbegrenzten Möglichkeiten in der modernen Intensivmedizin, des steigenden Kostendrucks im Gesundheitssystem und der zunehmend differenzierten ethischen Auseinandersetzung mit kritisch respiratorisch Kranken zwingend notwendig ist.

Interessanterweise steht seit kurzer Zeit eine äußerst lesenswerte Dissertationsschrift aus dem Institut für Geschichte und Ethik der Medizin der Johann Wolfgang Goethe-Universität Frankfurt am Main (Direktor: Prof. Dr. med. Dr. phil. Udo Benzenhöfer) zur Verfügung, in der Louise Reisner-Sénélar aus Dänemark das Lebenswerk von 
"Er (Prophet Elias) stieg auf das Bett und legte sich auf das Kind und legte seinen Mund auf des Kindes Mund und seine Augen auf dessen Augen und seine Hände auf dessen Hände und breitete sich so über ihn; da wurde des Kindes Leib warm ....; danach tat der Knabe seine Augen auf.

Zweites Buch der Könige 4, 34+35

Abb. 1 Erstbeschreibung einer Mund-zu-Mund-Beatmung in der Bibel.

Björn Ibsen und die dänische Polio-Epidemie 1952/53 skizziert, wobei einmalige Archivrecherchen und ein eigenes Interview mit Ibsen kurz vor seinem Tod die Kernelemente der Arbeit darstellen. Die Informationen zur Polio-Epidemie in diesem Artikel stammen im Wesentlichen aus dieser Arbeit, auf die an dieser Stelle verwiesen werden darf [1].

\section{Frühe Entwicklungen in der Beatmungsmedizin}

Die Erstbeschreibung einer Beatmung im Rahmen einer Wiederbelebung findet sich in der Bibel ( $\mathbf{A b b}$. 1). Danach hat es bis ins 16. Jahrhundert gedauert, bis die Beatmung wissenschaftlich untersucht worden ist ( Tab. 1). Das erste technische Gerät zur mechanischen Ventilation von Menschen wurde 1773 beschrieben und primär bei der Wiederbelebung eingesetzt. Wesentlich für die historische Entwicklung war die Erfindung und Weiterentwicklung des Tankventilators im 19. Jahrhundert, bei dessen Anwendung in der Regel der ganze Körper mit Ausnahme des Kopfes durch einen „Tank“ eingeschlossen wurde und so eine Negativ-Druck-Ventilation mittels manuell aufgebautem inspiratorischen Sog durchgeführt wurde. Die Kombination solcher Tankventilatoren mit Elektrizität erbrachte schließlich in der ersten Hälfte des 20. Jahrhundert automatisierte Respiratoren, die als „eiserne Lungen“ in die Geschichte eingegangen sind. Es gab zwar bereits in der 2. Hälfte des 19. Jahrhunderts erste Entwicklungen zur Laryngoskopie, Tracheotomie und Intubation, die im 20. Jahrhundert rasch weiterentwickelt wurden und durchaus auch während der Narkose zur Aufrechterhaltung der Ventilation zum Einsatz kamen. Einflussreiche Medizinerpersönlichkeiten waren jedoch durch Weiterentwicklungen der Negativ-DruckBeatmung, wie z.B. die Unterdruckkammer, die von Ferdinand Sauerbruch für die Thoraxchirurgie entwickelt wurde, maßgeblich daran beteiligt, dass die Positiv-Druck-Beatmung in der ersten Hälfte des 20. Jahrhunderts klinisch eine untergeordnete Rolle spielte. So waren die eisernen Lungen die ersten Respiratoren, die klinisch eine breite Anwendung fanden, insbesondere zur Behandlung der Poliomyelitis. Eine Zusammenstellung der historischen Meilensteine in der Entwicklung der Beatmungsmedizin bis zur Polio-Epidemie 1952 in Dänemark ist in $\bullet$ Tab. 1 gegeben. Eine detaillierte Übersicht über die Entwicklungen in der Beatmungsmedizin kann einer Dissertationsschrift entnommen werden, die unter der Anleitung von Prof. Dr. Wolfram Windisch (Abteilung Pneumologie der Albert-Ludwigs-Universität Freiburg) im Jahre 2008 entstanden ist [2].

\section{Bedeutung der Polio-Epidemie in Kopenhagen für die Entwicklung der außerklinischen Beatmung $\nabla$}

Im Jahre 1952 brachte die Poliomyelitis mit ihrer Epidemie eine Katastrophe von medizinhistorischer Tragweite nach Dänemark. Im Ausbruchsjahr 1952 wurden 5722 Poliomyelitis-Fälle in Dänemark registriert, fast die Hälfte davon hatte Atmungslähmungen; die Dunkelziffer an Poliomyelitis-Patienten dürfte aber noch höher liegen. In Kopenhagen konnten diese Patienten ausschließlich in dem für Infektionskrankheiten ausgerichteten Blegdams Hospital (500 Betten) behandelt werden. Entsprechend wurden alleine vom 24. Juli bis zum 3. Dezember 1952 insgesamt 2722 Poliomyelitis-Patienten behandelt. Der Umstand aber, dass lediglich sieben Respiratoren zur Verfügung standen (eine eiserne Lunge, sechs Kürass-Respiratoren), belegt eindeutig, dass die Klinik in keinem Fall für die Beatmung von täglich neuen Patienten

\begin{tabular}{|c|c|c|}
\hline Jahr & Entwicklung & Begründer \\
\hline ca. 560 v. Chr. & Erstbeschreibung einer Mund-zu-Mund-Beatmung & Altes Testament/2 Kön. \\
\hline ca. 1530 & Theorie der mechanischen Atemunterstützung & Paracelsus \\
\hline ca. 1543 & Rhythmisches Aufblasen von Tierlungen & Vesalius \\
\hline 1744 & Einführung der Mund-zu-Mund-Ventilation & John Fothergill \\
\hline 1773 & Erstes Gerät zur mechanischen Wiederbelebung & Alexander Johnston \\
\hline 1838 & Erstbeschreibung des Tankventilators & John Dalziel \\
\hline 1855 & Laryngoskopie & Manuel García \\
\hline 1869 & $\begin{array}{l}\text { Erste künstliche Beatmung eines Menschen zur Aufrechterhaltung } \\
\text { der Ventilation während einer Narkose }\end{array}$ & Friedrich Trendelenburg \\
\hline 1871 & Tracheostoma-Tubus mit Cuff & Friedrich Trendelenburg \\
\hline 1876 & Entwicklung der Spirophore & Eugéne J. Woillez \\
\hline 1876 & Erstentwurf eines Kürass-Respirator & Ignaz von Hauke \\
\hline 1878 & Orale endotracheale Intubation & William Mc Ewen \\
\hline 1904 & Unterdruckkammer für die Thoraxchirurgie & Ferdinand Sauerbruch \\
\hline 1905 & $\begin{array}{l}\text { Zylindrische Kopfkammer: CPAP am spontan atmenden Patienten } \\
\text { mittels handbetriebenem Kompressor }\end{array}$ & Ludolph Brauer \\
\hline 1928 & Erste Eiserne Lunge & Philip Drinker \\
\hline 1931 & Weiterentwicklung und Verbreitung der eisernen Lunge & John Haven Emerson \\
\hline 1932 & Rocking bed & FC Eve \\
\hline 1952 & $\begin{array}{l}\text { Poliomyelitis-Epidemie in Dänemark: } \\
\text { Positiv-Druck-Ventilation nach Tracheotomie bei respiratorischer } \\
\text { Insuffizienz, z. T. als Langzeitbeatmung }\end{array}$ & Björn Ibsen \\
\hline
\end{tabular}

Tab. 1 Meilensteine in der historischen Entwicklung der Beatmungsmedizin. 
vorbereitet war. Bei den schwersten Formen mit Bulbärparalyse und respiratorischer Insuffizienz bestand die Therapie bei den meisten Patienten somit lediglich in Tracheotomie und Sauerstoffinsufflation, allerdings mit einer Mortalität von über $90 \%$. So war das Behandlungsteam unter der Leitung von Henry Lassen gezwungen, nach schnellen Lösungen zu suchen und zu improvisieren.

Aus diesem Grund wurde Björn Ibsen (30.8.1915-7.8.2007) an die Klinik gerufen. Ibsen hatte nach ersten Erfahrungen als Narkosearzt in Jütland und am Rigshospital der Universitätsklinik in Kopenhagen Kenntnisse in der endotrachealen Intubation und der Beatmungsmedizin in Bosten/USA gesammelt und war nach innovativen Therapiekonzepten bei der Behandlung von Tetanus-Patienten, bei denen er Narkotika, Muskelrelaxantien und künstliche Beatmung kombinierte, empfohlen worden. Er war seit 1950 freiberuflich in Kopenhagen tätig.

Ibsen erkannte, dass das wesentliche Problem in der lähmungsbedingten Hypoventilation und nicht primär in der Virämie mit einem „cerebralen Befall“ lag. Nachdem er am 25.8.1952 seine Konzepte im Blegdams Hospital vorgestellt hatte und die Erkrankung an klinischen Fällen sowie bei Obduktionen vor Ort studiert hatte, wurde am 27. 8. 1952 die Patientin Vivi Ebert (1939-1971) mit schwerer Dyspnoe, Zyanose, Fieber, Diaphoresis und Paralyse aller vier Extremitäten in das Behandlungszimmer zur Therapie durch Ibsen geschoben. Nach Tracheotomie in Lokalanästhesie verschlechterte sich jedoch die klinische Situation mit zunehmender Unruhe und schließlich Bewusstlosigkeit. Nachdem Ibsen aber Thiopental verabreicht hatte und die Atemwege absaugen konnte, war eine manuelle Überdruckbeatmung über das Tracheostoma möglich.

Bereits acht Tage nach diesem eindrucksvollen klinischen Erfolg wurden drei neue Stationen mit je 35 Betten im Blegdams Hospital eingerichtet, auf der alle Poliomyelitis-Patienten mit respiratorischen Problemen von einem interdisziplinären Team behandelt und überwacht wurden. Die Mortalität konnte rasch von über $90 \%$ auf ca. $25 \%$ gesenkt werden. Allerdings wurde hierzu zusätzliche Hilfe in der Behandlung notwendig, da es bei einem Großteil der Patienten zur Langzeitbeatmung in der Regel manuell mittels Beatmungsbeutel über mehrere Wochen und Monate kam. So wurden auf dem Höhepunkt der Epidemie täglich 75 Patienten auf diese Weise beatmet, wobei 250 Medizinstudenten sowie zusätzlich 260 Krankenschwestern im 6-Stunden-Schichtdienst in die Behandlung dieser Patienten mit einbezogen wurden.

Vivi wurde bis Januar 1953 manuell beatmet, war dann bis 1955 komplett und danach intermittierend respiratorpflichtig und starb 1971 an einer Pneumonie. Allerdings wurde Vivi trotz Tetraplegie und Beatmungsabhängigkeit auf Drängen der Mutter und nach ausführlicher Einweisung in die Beatmungspflege nach Hause entlassen, was eindrucksvoll dokumentiert, dass die frühen 50er-Jahre des letzten Jahrhunderts nicht nur als Geburtsstunde der modernen Beatmungs- und Intensivmedizin betrachtet werden dürfen, sondern auch den Beginn der außerklinischen Langzeitbeatmung markieren.

\section{Entwicklung der außerklinischen Beatmung in Deutschland}

$\nabla$

Auch in Deutschland wurde die eiserne Lunge zur Behandlung der respiratorischen Insuffizienz verwendet, insbesondere bei Poliomyelitis-Patienten. Kriegsbedingt wurde die erste eiserne Lunge in Deutschland erst 1947 eingesetzt und damals in einer
Deutschen Werft aus dem Deckrohr eines Torpedos, dem Blasebalg einer Schmiede sowie aus Getriebeteilen eines Schiffs zusammengesetzt (Axel Dönhardt). Nach der Polio-Epidemie in Kopenhagen (s. oben) wurde jedoch ein unaufhaltsamer Prozess in Gang gesetzt, der in die Entwicklung der modernen Intensivmedizin mündete. Dabei wurden nach den Erfahrungen in Kopenhagen 1952 innerhalb weniger Jahre nicht nur in den USA, sondern auch in Europa und somit auch in Deutschland moderne Intensivrespiratoren zur Positiv-Druck-Beatmung in immer weiter verfeinerter Technik entwickelt, ein Prozess der bis heute andauert.

Allerdings war die Beatmung abgesehen von Einzelfällen auf die Intensivstation beschränkt. Langzeitbeatmung im außerklinischen Umfeld wurde weiterhin mit Negativ-Druck-Respiratoren (eiserne Lunge, Kürass-Respirator) durchgeführt. Das änderte sich jedoch Anfang der 80er-Jahre des letzten Jahrhunderts. Zunächst beschrieb Sullivan 1981 die Anwendung von Nasenmasken zur Behandlung des obstruktiven Schlafapnoesyndroms, während Yves Rideau 1983 erstmalig vergleichbare Masken zur nasalen Beatmung bei Patienten mit neuromuskulären Erkrankungen einsetzte.

In Deutschland ist die erste Maskenbeatmung 1985 in der Stiftung Pfennigparade München dokumentiert (Angelika Bockelbrink), an einem Zentrum für Langzeitbeatmung, an dem zuvor auch die Negativ-Druck-Respiratoren zum Einsatz gebracht wurden. Parallel dazu gab es eine wissenschaftliche Auseinandersetzung am Krankenhaus Bovenden-Lenglern bei Göttingen mit der Diagnostik und Therapie von Atempumpinsuffizienzen (Carl-Peter Criée und Gerhard Laier-Groeneveld). Nachdem 1982 das Konzept der erschöpften Atempumpe ausführlich von Charis Roussos beschrieben worden war, konnte in Lenglern erstmalig die Diagnostik solcher Störungen mit Hilfe der Mundverschlussdruck-Technik gelingen. Auf der Suche nach Behandlungsmöglichkeiten wurde der Kontakt nach Frankreich hergestellt, wo die nasale Maskenbeatmung bereits durchgeführt wurde. So konnten dann 1987 die ersten Patienten auch in Lenglern mittels nichtinvasiver Beatmung behandelt werden.

Es waren dann die Pioniere Criée und Laier-Groeneveld in Lenglern, die mit Kongresspräsentationen, dem Angebot zur Hospitation und mit der wissenschaftlichen Weiterentwicklung der nichtinvasiven Beatmung wesentlich zur Verbreitung der außerklinischen Beatmung beitrugen. An dieser Stelle darf auch auf die bereits oben erwähnte Dissertationsarbeit aus der Universitätsklinik Freiburg verwiesen werden, die die Entwicklung der nichtinvasiven Beatmung bis 2008 u.a. mit Hilfe von Interviews mit den Beteiligten der ersten Stunde detailliert skizziert hat.

Es folgte dann 1992 in Lenglern die Gründung des Arbeitskreises „Heim- und Langzeitbeatmung“ sowie 1994 daraus die Gründung der „Arbeitsgemeinschaft Heimbeatmung und Respiratorentwöhnung (AGH) e.V.“, in der Ärzte verschiedener Fachdisziplinen (insbesondere Pneumologen, Pädiater, Neurologen und Anästhesisten), nichtärztliche Berufsgruppen wie Krankenpflege und Physiotherapie u.a., Vertreter der Industrie sowie betroffene Patienten, Angehörige und Selbsthilfegruppen in interdisziplinärer Zusammenarbeit eine berufspolitische Verankerung der außerklinischen Beatmung in Deutschland bewirkt haben. Die AGH wurde 2010 umbenannt: „Deutsche Interdisziplinäre Gesellschaft für außerklinische Beatmung (DIGAB) e.V.“.

Spätestens mit Beginn des neuen Jahrtausends ist die außerklinische Beatmung zu einer weit verbreiteten und anerkannten Behandlungsmethode geworden, hinter der ein großer Industriezweig steht, auf die sich mittlerweile viele pneumologische Klini- 
Leitlinie

Nichtinvasive Beatmung als Therapie der akuten respiratorischen Insuffizienz [3]

Nichtinvasive und invasive Beatmung als Therapie der chronischen respiratorischen Insuffizienz [4]

Prolongierte Entwöhnung vom Respirator
Federführender Autor

B. Schönhofer

W. Windisch

B. Schönhofer
Erscheinungsjahr

2008

2010

in Bearbeitung
Tab. 2 Leitlinien zur Beatmungsmedizin, herausgegeben von der Deutschen Gesellschaft für Pneumologie und Beatmungsmedizin e. V. (DGP). ken in Deutschland spezialisiert haben und die in enger klinischer Beziehung zur Intensivmedizin steht. Dabei haben die intensivmedizinischen Behandlungserfolge der letzten Jahre zwar die Prognose auf der Intensivstation verbessert, viele vor allen Dingen polymorbide Patienten haben jedoch erhebliche Schwierigkeiten bei der Entwöhnung (Weaning) vom Respirator und werden schließlich mittels invasiver (Tracheostoma) oder nichtinvasiver (Gesichtsmaske) Beatmung in die außerklinische Beatmung übergeleitet. Dieser Bereich der Medizin erfordert neben einer spezialisierten und qualifizierten außerklinischen Beatmungspflege auch die professionelle Spezialisierung der Krankenversorgung innerhalb der Klinik.

Vor diesem Hintergrund hatte sich auch die „Deutsche Gesellschaft für Pneumologie (DGP)“ intensiv mit dem Thema der Beatmungsmedizin auseinandergesetzt. Nach einer bewegenden Hauptmitgliederversammlung mit Abstimmung zur Satzungsänderung initiiert durch den damaligen Präsidenten Dieter Köhler wurde die „Deutsche Gesellschaft für Pneumologie (DGP)“ 2006 in die „Deutsche Gesellschaft für Pneumologie und Beatmungsmedizin (DGP)“ umbenannt. Dies unterstreicht zweifellos die heutige berufspolitische Bedeutung der Beatmungsmedizin sowie auch den Stellenwert des Fachs Pneumologie für die Beatmungsmedizin.

Heute beschäftigen sich zwei Fachgesellschaften führend mit der nichtinvasiven Beatmung, mit der außerklinischen Beatmung sowie mit dem Weaning: die DIGAB und die DGP. Entsprechend werden seit 1993 Jahreskongresse zu diesen Themengebieten durchgeführt, die zunächst alleine von der DIGAB ausgerichtet wurden, seit 2006 aber gemeinsam durch die DIGAB und die DGP organisiert werden. Zudem hat die DGP in enger Zusammenarbeit mit der DIGAB und anderen Fachgesellschaften drei wissenschaftliche Leitlinienprojekte unter dem Dach der Arbeitsgemeinschaft der Wissenschaftlichen Medizinischen Fachgesellschaften e.V. (AWMF) herausgegeben ( $\bullet$ Tab. 2).

\section{Epidemiologie und Zukunft der außerklinischen Beatmung in Deutschland}

Epidemiologische Daten aus Europa von 2001 zeigen eine Prävalenz von 6,6 außerklinischen Beatmungen pro 100000 Einwohner, allerdings mit einer erheblichen Streuung innerhalb der verschiedenen europäischen Länder, wobei die Daten für Deutschland eine Prävalenz von 6,5 außerklinischen Beatmungen pro 100000 Einwohner ergeben [5]. Tatsächlich ist diese Zahl jedoch für die heutige Situation in Deutschland deutlich unterschätzt, da nur etablierte Beatmungszentren in der Erhebung berücksichtigt wurden, die Antwortquote für Deutschland (alle Länder) lediglich 41 (62)\% betrug und die Prävalenz in den letzten Jahren weiter gestiegen ist.

Während zudem noch vor wenigen Jahrzehnten Patienten mit neuromuskulären oder thorakal-restriktiven Erkrankungen die Hauptindikationen zur außerklinischen Beatmung stellten, sind es heute vor allen Dingen ältere, komplexe und polymorbide Patienten mit chronisch obstruktiver Lungenerkrankung, Adipositas-Hypoventilations-Syndrom oder solche nach erfolglosem Weaning. Dabei wird in der Zukunft eine weitere Zunahme an Langzeit-Beatmungsfällen erwartet. Dies belegt deutlich, dass die Epidemiologie innerhalb kurzer Zeit einem Änderungsprozess unterlegen ist. Die DIGAB hat daher ein Projekt gestartet, das zukünftig über den Aufbau einer nationalen Datenbank erlauben wird, genauere epidemiologische Daten im Zeitverlauf zu erfassen. Zudem hat die DGP die Initiative WeanNet (Leitung: Bernd Schönhofer) ins Leben gerufen, die zwar vorrangig auch das Ziel der Akkreditierung von Weaning-Zentren hat, um einen Wildwuchs von solchen Zentren ohne gesicherte Qualitätsstandards zu vermeiden. Zusätzlich wird aber auch hier als wesentliches Ziel der Aufbau einer Datenbank angestrebt, was zukünftig weitere epidemiologische Auswertungen zulässt.

Die Erfassung epidemiologischer Daten ist Vorraussetzung für eine suffiziente Versorgungsforschung in dem Bereich der außerklinischen Beatmung. Es ist zudem zukünftig auch die Aufgabe der Fachgesellschaften und der Berufspolitik, Zentren für diese spezialisierte Medizin zu definieren, die notwendigen Infrastrukturen zu schaffen, die für die aufwendige Logistik der Versorgung außerklinisch Beatmeter notwendig sind, sowie schließlich Qualitätsstandards für die pflegerische und ärztliche Behandlung von Beatmungspatienten im häuslichen Umfeld zu erstellen, um der stetig wachsenden Zahl von schwer kranken Patienten mit der Notwendigkeit zur Langzeitbeatmung gerecht zu werden.

\section{Interessenkonflikte \\ $\nabla$}

Der Autor gibt an, dass kein Interessenkonflikt besteht.

\section{Literatur}

1 Reisner-Sénélar L. Der dänische Anästhesist Björn Ibsen - ein Pionier der Langzeitbeatmung über die oberen Luftwegen [Dissertation]. Frankfurt am Main: Johann-Wolfgang-Goethe-Universität, 2009; http://publikationen.ub.uni-frankfurt.de/volltexte/2009/7318/

2 Stuke C. Die historische Entwicklung der nichtinvasiven Positiv-Druck Ventilation in Deutschland bis 2008 [Dissertation]. Freiburg i. Breisgau: Albert-Ludwigs-Universität, 2009; http://www.freidok.uni-freiburg.de/volltexte/6493/

3 Schönhofer B, Kuhlen R, Neumann et al. Nichtinvasive Beatmung als Therapie der akuten respiratorischen Insuffizienz - S3-Leitlinie herausgegeben von der Deutschen Gesellschaft für Pneumologie und Beatmungsmedizin. Pneumologie 2008; 62: 449-479

4 Windisch W, Brambring J, Budweiser $S$ et al. Nichtinvasive und invasive Beatmung als Therapie der chronischen respiratorischen Insuffizienz. S2-Leitlinie herausgegeben von der Deutschen Gesellschaft für Pneumologie und Beatmungsmedizin e.V. Pneumologie 2010; 64: $207-$ 240

5 Lloyd-Owen SJ, Donaldson GC, Ambrosino $N$ et al. Patterns of home mechanical ventilation use in Europe: results from the Eurovent survey. Eur Respir J. 2005; 25: 1025-1031 